\title{
Temperature and heat stress in a concrete column at early ages
}

\author{
P. F. Siew T. Puapansawat Y. H. Wu*
}

(Received 17 September, 2001; revised 12 June, 2002)

\begin{abstract}
The temperature development and the stress generated in a cylindrical column of concrete are investigated. The problem is essentially two-dimensional, and is solved using a finite element package. Adiabatic temperature data from four different concrete mixes are used and the influences on the temperature and stress development due to the use of silica fumes or slag in the mixes are discussed.
\end{abstract}

\section{Contents}

\section{Introduction}

*Department of Mathematics \& Statistics, Curtin University of Technology, Western Australia. mailto:pfsiew@maths.curtin.edu.au

${ }^{0}$ See http://anziamj . austms.org.au/V44/CTAC2001/Siew for this article, (C) Austral. Mathematical Soc. 2003. Published 1 April 2003. ISSN 1446-8735 
2 Formulation of the problem

2.1 Boundary conditions and parameters . . . . . C C710

3 The temperature development

C711

4 The thermal stress

C716

4.1 The compressive and splitting tensile strength . .

5 Discussion

C718

References

C720

\section{Introduction}

The prediction of the temperature and stress distributions in hardening concrete is of great interest to designers and contractors. This is because the hydration of cement is an exothermic process, and the generation of heat may lead to very early onset of thermal cracks in the absence of any loading. Depending on the geometry of the structure, and the type of concrete mix used, the temperature rise at the centre of the structure may range from $30^{\circ} \mathrm{C}$ to $70^{\circ} \mathrm{C}[9,1$, e.g.]. The concrete may also gain heat from the external environment, although this is difficult to monitor over a period of many days. Huang [8] reported that the non-uniform thermal deformations due to the difference between the inner and outer parts of a large structure led to early surface cracks, since the tensile strength of the concrete was still developing; whereas inner cracks were more likely to occur as the temperature cooled. Any analysis of the temperature development should be accompanied by an analysis of the stresses generated in the structure. The task is often made more difficult because of the variability in the composition of aggregates in the mix. 
This paper presents the analysis of the temperature development in a cylindrical concrete column, together with an analysis of the stresses generated in the structure as the cement hydrates. We assume that the aggregates in the mixture are small in size compared to the overall dimensions of the structure, so that the cement is regarded as a homogeneous material. Thus the problem becomes essentially two-dimensional and is easily solved using a finite element package.

\section{Formulation of the problem}

Consider a cylindrical column of fresh concrete with a radius of 0.5 metres and height 2 metres, sitting on a base of old concrete. The fresh concrete is surrounded by formwork (assumed to be plywood) on the sides, with insulating material over the top surface, both of thickness 0.02 metres. The temperature distribution in the concrete is governed by the equation

$$
\frac{\partial}{\partial r}\left(k r \frac{\partial T}{\partial r}\right)+\frac{\partial}{\partial z}\left(k r \frac{\partial T}{\partial z}\right)+r \dot{q}=\rho c r \frac{\partial T}{\partial t},
$$

where $k$ is the thermal conductivity, $T$ denotes the temperature, $\dot{q}$ denotes the rate of heat generated inside the body, $\rho$ is the density of concrete, $c$ is the specific heat, and $t$ is the time. Cylindrical coordinates $(r, \theta, z)$ with $z$ positive upward, is taken with origin at the bottom of the cylindrical column, and $r$ is the radial distance from the axis of the cylinder. Measurements of thermal conductivity of concrete mixes are difficult to make. As reported in [9], there seems to be no consistent results regarding the thermal conductivity of fresh concrete. Some investigators maintain that it varies by up to $30 \%$ as the concrete matures from 6 hours to 7 days, whereas others maintain that it does not change with age. On the other hand, 
Gibbon and Ballim [7] reported that the thermal conductivity of mortar, made with various sands or binders, varies considerably during the first 3 days, but the calculated thermal conductivity of the concrete mix is practically constant over time, although different for different mixes. The measurement of the specific heat of hardening concrete is equally difficult. Most authors report a decrease in the specific heat during hardening, but there is no agreement concerning the size of the decrease. Schutter and Taerwe [6] presented some data on the diffusivity versus the degree of hydration, and regression analysis showed that a linear fit to the data gives the diffusivity variation at about $5 \%$ over the hardening period. Hence we assume a constant thermal conductivity and diffusivity for our investigation. Assuming that the product $\rho c$ is a fixed quantity, the temperature distribution in the concrete is completely specified once $\dot{q}$ and $k$ are known.

Although concrete is a brittle material that may exhibit some viscoelastic properties, most studies have tended to regard it as an elastic material up to the point where the material has yielded under some form of loading. With this assumption, and neglecting body forces, the stress distribution within the column is governed by (see for example, Benham and Hoyle [3])

$$
\begin{aligned}
\frac{\partial \sigma_{r r}}{\partial r}+\frac{\partial \sigma_{r z}}{\partial z}+\frac{\sigma_{r r}-\sigma_{\theta \theta}}{r} & =0, \\
\frac{\partial \sigma_{r z}}{\partial r}+\frac{\partial \sigma_{z z}}{\partial z}+\frac{\sigma_{r z}}{r} & =0,
\end{aligned}
$$

where $\sigma_{r r}, \sigma_{\theta \theta}, \sigma_{z z}$ and $\sigma_{r z}$ are the radial, hoop, axial and shear stresses respectively. Letting $u$ and $w$ denote the radial and axial displacements, then the equation linking the vector $U=[u, w]^{\top}$ to 
the stresses is

$$
S=C D U-\beta \Phi\left[\begin{array}{l}
1 \\
1 \\
1 \\
0
\end{array}\right]
$$

where

$$
\begin{aligned}
S & =\left[\begin{array}{c}
\sigma_{r r} \\
\sigma_{\theta \theta} \\
\sigma_{z z} \\
\sigma_{r z}
\end{array}\right], \quad C=\left[\begin{array}{cccc}
\lambda+2 \mu & \lambda & \lambda & 0 \\
\lambda & \lambda+2 \mu & \lambda & 0 \\
\lambda & \lambda & \lambda+2 \mu & 0 \\
0 & 0 & 0 & \mu
\end{array}\right], \\
\text { and } \quad D & =\left[\begin{array}{cc}
\partial / \partial r & 0 \\
1 / r & 0 \\
0 & \partial / \partial z \\
\partial / \partial z & \partial / \partial r
\end{array}\right] .
\end{aligned}
$$

Here $\lambda=\nu E /[(1+\nu)(1-2 \nu)], \beta=\alpha(3 \lambda-2 \mu), \Phi$ is the change in temperature, $\nu$ is Poisson's ratio, $E$ is the Young's modulus, $\mu$ is the modulus of rigidity and $\alpha$ is the coefficient of linear thermal expansion.

We assume that the ground temperature is the same as the ambient temperature $T_{\infty}$, and that the concrete is mixed and poured at time $t=0$. Defining $\Phi=T-T_{\infty}$, and rewriting the coordinates $(r, z)$ as $\left(x_{1}, x_{2}\right)$, equation (1) becomes

$$
\frac{\partial}{\partial x_{i}}\left(x_{1} k \frac{\partial \Phi}{\partial x_{i}}\right)+x_{1} \dot{q}=x_{1} \rho c \frac{\partial \Phi}{\partial t},
$$

whereas, substituting in terms of the displacements, (2) becomes

$$
\lambda \frac{\partial u_{j j}}{\partial x_{i}}+\lambda \frac{\partial}{\partial x_{i}} \frac{u_{1}}{x_{1}}-\beta \frac{\partial \Phi}{\partial x_{i}}+2 \mu \frac{\partial}{\partial x_{j}} u_{i j}+B_{i}=0,
$$


TABLE 1: material parameters.

\begin{tabular}{|c|ccccc|}
\hline & $\begin{array}{c}k \\
{\left[\mathrm{~kJ} /\left(\mathrm{h} \mathrm{m}^{\circ} \mathrm{C}\right)\right]}\end{array}$ & $\begin{array}{c}c \\
{\left[\mathrm{~kJ} /\left(\mathrm{kg}^{\circ} \mathrm{C}\right]\right.}\end{array}$ & $\begin{array}{c}\rho \\
{\left[\mathrm{kg} / \mathrm{m}^{3}\right]}\end{array}$ & $\alpha$ & $\nu$ \\
\hline Concrete & 7.2 & 1.006 & 2370 & $1.04 \times 10^{-5}$ & 0.2 \\
Plywood & 0.432 & 1.213 & 545 & $4 \times 10^{-6}$ & 0.17 \\
\hline
\end{tabular}

where

$$
\begin{aligned}
u_{i j} & =\frac{1}{2}\left(\frac{\partial u_{i}}{\partial x_{j}}+\frac{\partial u_{j}}{\partial x_{i}}\right) \\
B_{i} & =\left[2 \mu \frac{\partial}{\partial x_{1}} \frac{u_{1}}{x_{1}}, \mu\left(\frac{\partial}{\partial x_{2}} \frac{u_{1}}{x_{1}}+\frac{1}{x_{1}} \frac{\partial u_{2}}{\partial x_{1}}\right)\right]^{\top},
\end{aligned}
$$

$u_{1}=u, u_{2}=w$, and a repeated suffix denotes summation.

\subsection{Boundary conditions and parameters}

The parameters used in this study, given in Table 1 , are obtained from various sources $[1,9,10$, e.g.]. The thermal diffusivity, $k /(\rho c)$, of the concrete mix is therefore assumed to be $0.00302 \mathrm{~m}^{2} / \mathrm{hr}$. The convection coefficient, which depends on the size of the concrete column, is taken to be $29.88 \mathrm{~kJ} /\left(\mathrm{h} \mathrm{m}^{2}{ }^{\circ} \mathrm{C}\right.$ ) (cf. [9]). The Young's modulus

$$
E=3.385 \times 10^{-5} w^{2.5}\left(f_{c}\right)^{0.325} \mathrm{MPa},
$$

where $f_{c}$ and $w$ are the compressive strength and unit weight of the concrete (see [10]).

We assume that the insulation material has the same thermal conductivity and expansion coefficient as plywood. At internal boundaries (between concrete and formwork or insulation), we require that the heat flux be continuous, whereas on the air/material 
interface, we require $-k_{s} \partial \Phi / \partial n=h_{a} \Phi$, where $h_{a}$ is the convective heat transfer coefficient, and $n$ is the unit outward pointing normal to the surface, which has thermal conductivity $k_{s}$. In the finite element formulation, see from (4) that the heat flux (multiplied by $x_{1}$ ) forms the boundary integrand. See in equation (5) that the boundary integrand gives the stress. Assuming that the contact between the concrete and the formwork is maintained throughout, the stress is continuous across internal boundaries and is taken to be zero on the air/material interface. The finite element package FASTFLO [5, e.g.], is used in the numerical simulation. Equation (4) is solved using a time stepping algorithm. At the end of each time period, the temperature is fed into the displacement equations in (5), to obtain the displacement vector. Then using the mesh mapping facility in the package, the coordinates of each mesh point are adjusted and the process is repeated for the next time step.

\section{The temperature development}

The adiabatic temperature rise in a sample of each of four different concrete mixes were obtained over a period of 150 hours. Each of the four mixes have a design strength of $50 \mathrm{MPa}$ (that is the compressive cylinder strength), although the actual strength after 28 days is closer to $65 \mathrm{MPa}$. Mix I uses ordinary Portland cement (OPC). Mix II is OPC with $8 \%$ silica fume added (OPC/CSF). Mix III is a low heat (LH) mix with $65 \%$ slag and Mix IV is a low heat mix with $5 \%$ silica fume ( $\mathrm{LH} / \mathrm{CSF})$ and $62 \%$ slag. Figure 1 gives the rate of heat generated [in $\left.\mathrm{kJ} /\left(\mathrm{hr}{ }^{\circ} \mathrm{C}\right)\right]$ per unit volume calculated from the adiabatic temperature release for each mix.

Using the usual one parameter approximation for the time deriva- 


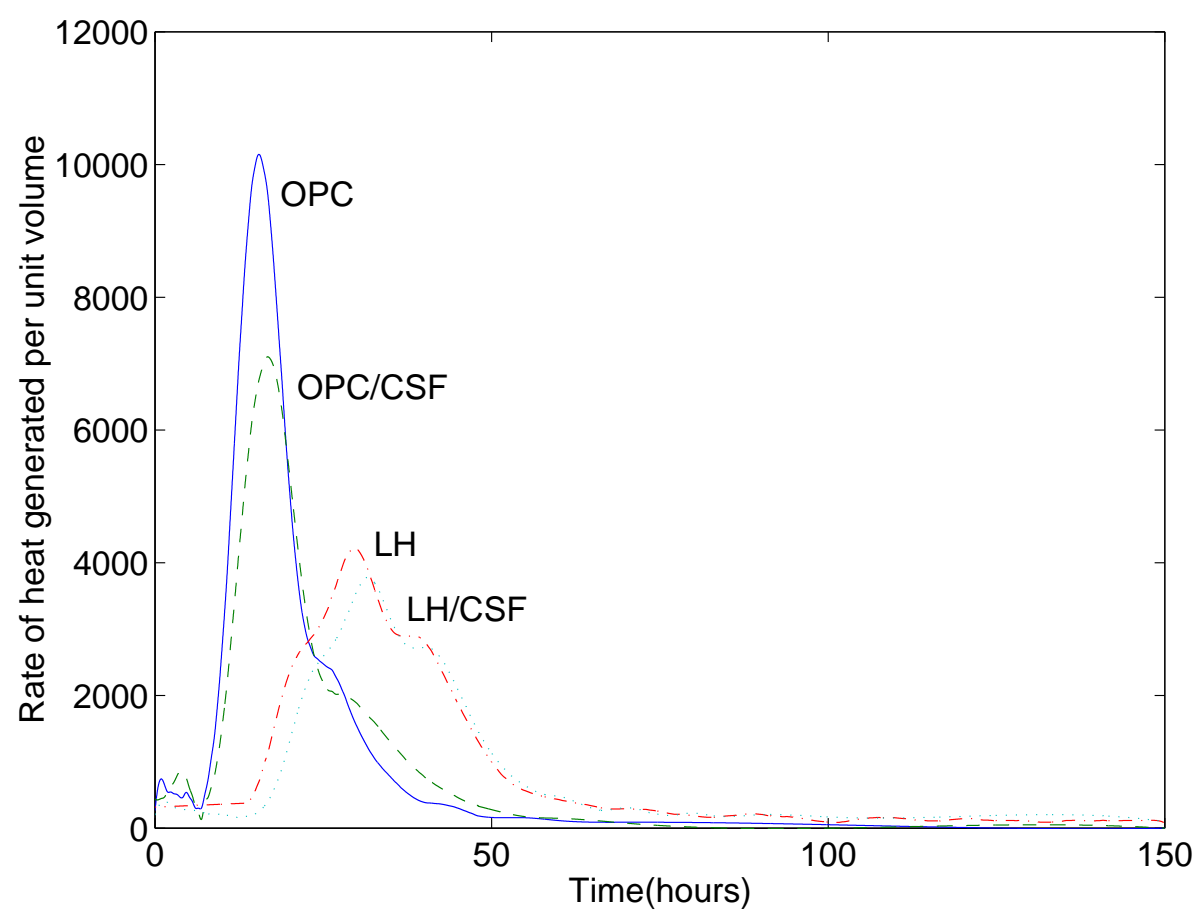

FiguRE 1: Rate of generation of heat for the four mixes 


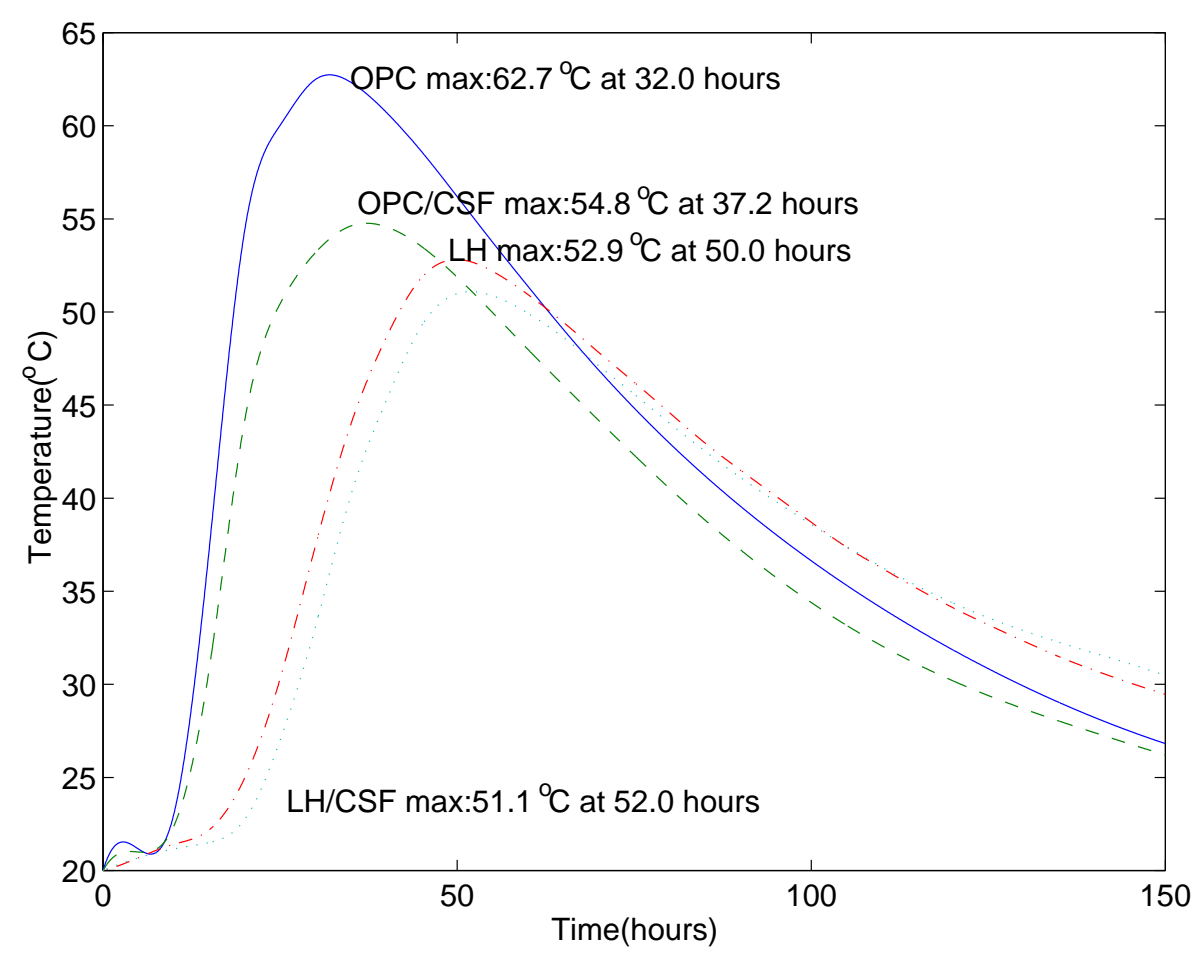

Figure 2: Temperature profile as a function of time

tive, we rewrite (4) as

$$
\begin{aligned}
x_{1} \rho c \Phi_{n+1}= & x_{1} \rho c \Phi_{n}+k \theta_{s} \Delta t \nabla \cdot\left(x_{1} \nabla \Phi_{n+1}\right) \\
& +k\left(1-\theta_{s}\right) \Delta t \nabla \cdot\left(x_{1} \nabla \Phi_{n}\right)+x_{1} Q_{n},
\end{aligned}
$$

where $Q_{n}$ is the heat generated at the $n$th time step. Here $\theta_{s}=\frac{1}{2}$ corresponds to the Crank Nicholson scheme, whereas $\theta_{s}=\frac{2}{3}$ corresponds to the Galerkin scheme. The Galerkin scheme is used in the simulation. Figure 2 gives the plots of the temperature rises in the concrete column for the four different mixes, as a function of time, at the point on the axis at height 1.1 metres from the bottom. This is also the location where the maximum temperature in the column is 
attained. The highest increase is obtained with OPC. The addition of silica fumes reduces the maximum temperature reached by $13 \%$, whereas the use of slag decreases it by $16 \%$. The use of low heat concrete with silica fumes not only decreases the maximum temperatures by $19 \%$, but also produces the lowest rate of temperature increase. Since the rate of increase of the temperature is believed to have a significant influence on the cracking of concrete, the low heat with silica fume mixture appears to give the best performance in this regard. The distribution of the temperature in the column, at the time when the maximum temperature is reached, is given in the contour plots in the $r z$ plane, in Figure 3. Note that the thermal conductivity will influence the temperature distribution. Some authors have used a value for $k$ for matured concrete as high as $10.8 \mathrm{~kJ} /\left(\mathrm{h} \mathrm{m}^{\circ} \mathrm{C}\right)$. This means the heat flux is increased and will result in lower temperatures. For example, if we use $k=9$, the maximum temperature for $\mathrm{OPC}$ is reduced to $61.7^{\circ} \mathrm{C}$, attained at the earlier time of 31.5 hours. Several investigators have suggested (see for example, Bamforth [2]) that the temperature differentials between mid-height to the top or side of a concrete block should be made as small as possible in order to minimise the possibility of cracking due to thermal stress. As a rule of thumb Bamforth used $20^{\circ} \mathrm{C}$ as the limiting differential. The recommended temperature differentials for mass concrete pours depend on the aggregates used in the mix, and range from about $20^{\circ} \mathrm{C}$ for gravel to about $28^{\circ} \mathrm{C}$ for granite. These figures are based on experimental observations, but as mentioned in [2], it does not mean that the concrete will crack when these figures are exceeded, because it is the maximum strain that the concrete can tolerate that is crucial. In our simulation, the differentials for all mixes exceed $20^{\circ} \mathrm{C}$ along the bottom outside surface of the concrete. This suggests that all of the mixes may fail, and it is therefore important to consider the stresses in the concrete as the cement hydrates. 

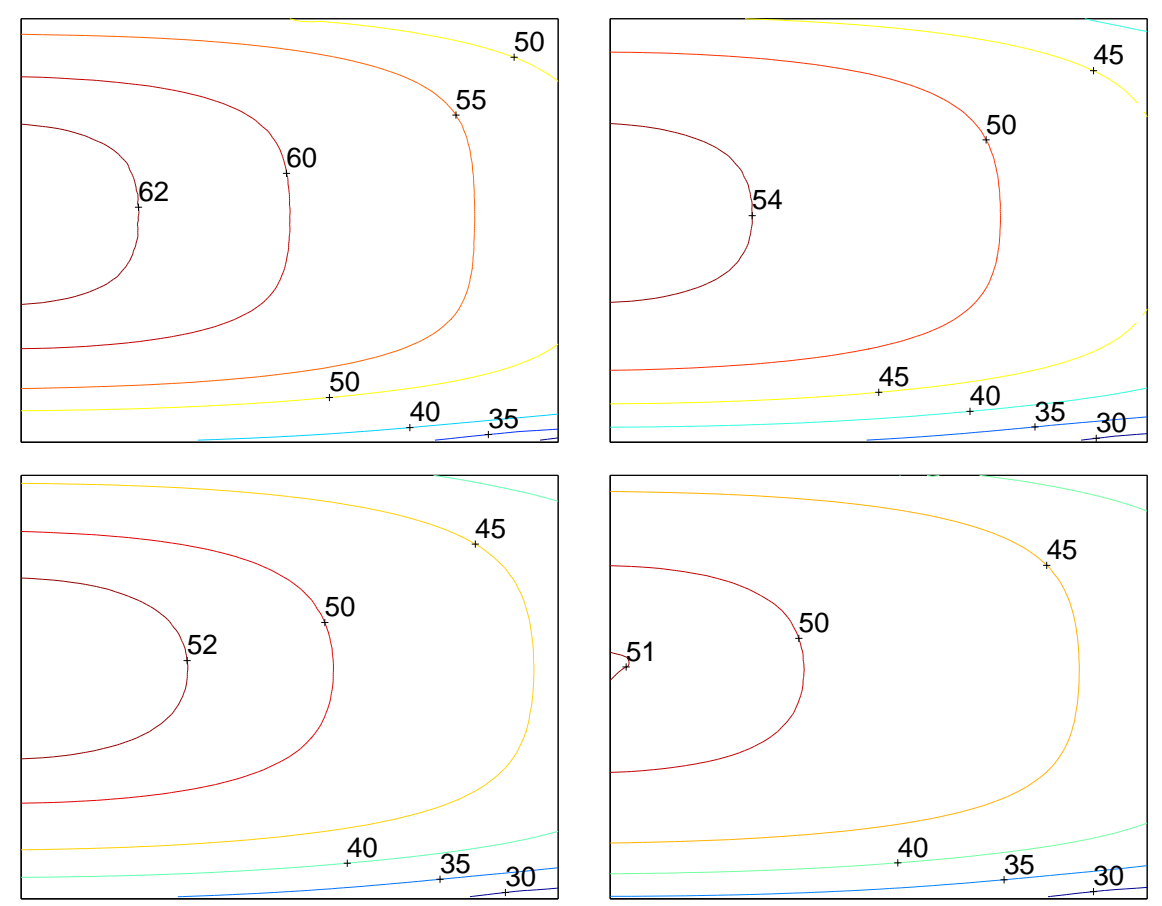

Figure 3: Temperature contours in the $r z$ plane for the concrete column (from top left, OPC, OPC/CSF, LH and LH/CSF). [Dimensions not to scale] 


\section{The thermal stress}

From the solution to equation (5), the stress components are obtained using equation (3). The principal stress components are easily given by the solution to the equation $S^{3}-I_{1} S^{2}+I_{2} S-I_{3}=0$, where $I_{1}, I_{2}$ and $I_{3}$ are the first three invariants of the stress tensor [12]. One of the principal stresses is the hoop component $\left(\sigma_{\theta \theta}\right)$, which we will denote by $\sigma_{1}$, the other two,

$$
\sigma_{2}, \sigma_{3}=\frac{1}{2}\left[\sigma_{r r}+\sigma_{z z} \pm \sqrt{\left(\sigma_{r r}-\sigma_{z z}\right)^{2}+4 \sigma_{r z}^{2}}\right],
$$

where the plus sign corresponds to $\sigma_{2}$.

The formwork around the cylindrical column may be restrained in some fashion to keep its shape, especially in the earlier periods after the pour. We therefore impose the condition that $u_{1}=0$ on the solid/air interface around the curved boundary. In the numerical simulation, the stress state of the concrete is in compression as the temperature in the column increases. This is true up to about 30 hours after the pour. In all four mixes, the highest compressive stress is generated by the hoop component $\sigma_{1}$. The time taken to reach the maximum occurs between 0.1 and 0.2 hours before the maximum temperature is attained. The maximum compressive stresses reached in the four mixes are respectively $26 \%, 21 \%, 22 \%$ and $19 \%$ of the 28 day compressive strength for OPC, OPC/CSF, LH, and LH/CSF. As the material cools, more regions in the concrete are brought into a state of tension. The maximum tensile stresses reached are 2.0, 1.6, 1.5, and $1.4 \mathrm{MPa}$ for the four mixes, in the same order as before. These maxima are attained typically about an hour after the maximum temperature in the mix has been reached. The stresses generated in the concrete column are certainly not excessive. However, the compressive and tensile strengths of young concrete develop at rates depending on the degree of hydration of the con- 
crete. It is therefore necessary to consider these rates of development and the stresses that are generated as the concrete matures.

\subsection{The compressive and splitting tensile strength}

The compressive and tensile strengths in fresh concrete develops over time and hence it is necessary to keep track of the stresses generated as a consequence of the heat released due to hydration. Estimates of the compressive strength of the four mixes were given in terms of piecewise linear fits in Chen et al. [4] over a period of 28 days. Since the temperature data that is provided only goes for 150 hours (slightly beyond 6 days) the approximate linear fit over the period from 1 day to 7.5 days gives the compressive strengths for OPC, OPC/CSF LH and LH/CSF respectively as

$$
\begin{aligned}
& f_{c, \mathrm{OPC}}=\frac{118}{13}+\frac{64}{13} t_{d}, \quad f_{c, \mathrm{OPC} / \mathrm{CSF}}=\frac{82}{13}+\frac{74}{13} t_{d}, \\
& f_{c, \mathrm{LH}}=\frac{62}{15} t_{d}, \quad f_{c, \mathrm{LH} / \mathrm{CSF}}=\frac{44}{15} t_{d},
\end{aligned}
$$

where $t_{d}$ denotes the time in days and, where necessary, a linear increase is assumed from $t_{d}=0$ to $t_{d}=1$. In the case of $\mathrm{LH}$ and LH/CSF, slightly higher compressive strength were taken for the fit here than in [4], where $f_{c, \mathrm{LH}}$ and $f_{c, \mathrm{LH} / \mathrm{CSF}}$ appears to be practically equal to zero for $t_{d}$ between 0 and 1 . The concrete may be expected to fail when the (principal) compressive stress generated by the release of heat exceeds its compressive strength.

Similarly, using the Rankine criterion, the concrete is expected to crack when the tensile stress exceeds the tensile strength of the concrete. One problem with this criterion is the difficulty in defining 
the splitting tensile stress $f_{s p}$. The reader is referred to [10] for a discussion of the various formulae in use. We use

$$
f_{s p}=c_{f} \sqrt{f_{c}}
$$

where $c_{f}=0.56$. This equation was proposed in the $1999 \mathrm{ACI}$ Committee 318 Report. In fact, in the 1992 ACI Committee 363 Report, $c_{f}=0.59$ was proposed for high strength concrete.

A direct measurement of the tensile strength $\hat{f}_{t}$ for hardening concrete is given in [11] as $\hat{f}_{t} \approx\left(\tilde{\alpha}-\tilde{\alpha}_{0}\right) /\left(1-\tilde{\alpha}_{0}\right)$. Here $\hat{f}_{t}$ has been normalized to equal 1 when the concrete has fully hydrated, $\tilde{\alpha}$ is a function of temperature and is the degree of hydration, measured as the ratio of the heat generated to that point in time over the total heat of hydration, and $\tilde{\alpha}_{0}$ is the initial degree of hydration when the concrete is poured. This equation may be compared with the linear equation given in [8], when $\tilde{\alpha}_{0}$ is small. Assuming the tensile strength is close to the splitting tensile strength, we derive another alternative equation for $f_{s p}$. Since we have assumed that the pour temperature is equal to the ambient temperature, $f_{s p} \approx 0.59 \tilde{\alpha} \sqrt{f_{c, 28}}$ may be used as another equation for the splitting tensile strength, where $f_{c, 28}$ is the 28 day compressive strength of the concrete.

The slowest rate of increase in the splitting tensile stress is given by (8), with $c_{f}=0.56$, which is the one we use in this study. In the numerical simulation, the tensile and compressive stresses in the concrete are checked against the tensile and compressive strengths as given by (8) and (7) at the end of each time step.

\section{Discussion}

The maximum compressive stress generated in the concrete can be plotted against time starting from the time the concrete is poured. 
This curve is then superimposed on the appropriate curve given by (7). In each of the cases of OPC, LH and LH/CSF, the maximum compressive stress generated exceeds the developing compressive strength for the particular mixture 16.9, 24.9 and 25.8 hours, respectively, after the pour. This occurs near the axis of the column about 1.3 metres from the base. For OPC/CSF, the two curves appear to touch at one point. Given the approximate nature of the curves given by (7), the mixture with $8 \%$ silica fume may not have failed. On the other hand, except for LH/CSF, the tensile stress generated in the other three mixes never exceeds the developing tensile strength of the concrete, as given by (8). If we use $c_{f}=0.59$ in (8), then none of the four mixes will develop tensile stresses that exceed the splitting tensile stress curve.

Based on the parameters used in sections 3 and 4, and comparing the performance of the four mixes, see that while LH/CSF concrete generates the least heat, at a slower rate, its compressive strength also develops much slower and hence it has a tendency to fail. The addition of $8 \%$ silica fume to OPC has the effect of lowering the heat generated, but at the same time, does not reduce the rate of gain of compressive strength. This makes it the most suitable mix in a general sense. One other factor, which has not been taken into account in this study, is that the development of the temperature in the concrete is actually not adiabatic; there is usually some heat loss to the environment. This situation is not modelled due to the lack of data for modelling the effects of radiation or wind chill. In our simulation, the formwork is in place over the entire period under consideration and we assume no variation in the ambient temperature. In the real situation, the condition $u_{1}=0$ should be relaxed as we expect there will be some free movement of the vertical part of the formwork containing the concrete mixture.

In conclusion, microcracks will usually appear in any mass con- 
crete pour, although it is difficult to see them. Cracks at boundaries between the fresh concrete and other material are usually observed, and these cracks are important if they can spread into the interior of the concrete. Modelling can determine some of the parameters that are important in minimising the occurrence of cracks. Concrete properties are influenced by the aggregates used in each mix, and depending on location, there can be a lot of variations; for example, crushed rock is usually used in Western Australia, while elsewhere, gravel or limestones may be used. Empirical laws are therefore to be used with a lot of care, especially for hardening concrete at early ages where many of the parameters have not yet reached their final values.

Acknowledgement: The authors are grateful to Mr Phillip Chen, for the adiabatic temperature data used in this paper.

\section{References}

[1] E. Ayotte, B. Massicotte, J. Houde and V. Gocevski, "Modeling the thermal stresses at early ages in a concrete monolith", ACI Materials Journal 94 1994, 577-587. C706, C710

[2] P. B. Bamforth, "In situ measurement of the effect of partial Portland cement replacement using either fly ash or ground granulated blast-furnace slag on the performance of mass concrete", Proc. Instn. Civ. Engrs, Part 2, 69 1980, 777-800. C714

[3] P. P. Benham and R. Hoyle, Thermal Stress, (Sir Isaac Pitman \& Sons Ltd, 1964). C708 
[4] P. Chen, F. Papworth, P. Trinder and A. Peek, Low heat concrete, private communication, from a talk delivered to the Concrete Institute of Australia, Perth, Western Australia, 10pp. C717

[5] Commonwealth Scientific and Industrial Research Organisation (CSIRO) 1999, Fastflo tutorial guide, version 3, 378pp. C711

[6] G. De Schutter and L. Taerwe, "Specific heat and thermal diffusivity of hardening concrete" Magazine of Concrete Research 471995 203-208. C708

[7] G. J. Gibbon and Y. Ballim, "Determination of the thermal conductivity of concrete during the early stages of hydration", Magazine of Concrete Research 50 1998, 229-235. C708

[8] C.-X. Huang, "The three dimensional modelling of thermal cracks in concrete structure", Materials and Structures $\mathbf{3 2}$ 1999, 673-678. C706, C718

[9] A. A. Khan, W. D. Cook and D. Mitchell, "Thermal properties and transient thermal analysis of structural members during hydration", ACI Materials Journal 95 1998, 293-302. C706, C707, C710

[10] A. Mokhtarzadeh and C. French, "Mechanical properties of high-strength concrete with consideration for precast applications", ACI Materials Journal 97 2000, 136-147. C710, C718

[11] F. S. Rostasy, A. Gutsch and M. Laube, "Creep and relaxation of concrete at early ages - experiments and mathematical modeling", in Creep and shrinkage of concrete Proc. 5th Intern. RILEM Symp., 1993, 453-458. C718 
[12] S. P. Timoshenko and J. N. Goodier, Theory of elasticity, 3rd ed. (McGraw-Hill, 1970) C716 